\title{
The impact of chronic in vivo glucocorticoid excess on the functional characteristics of human skin fibroblasts obtained from patients with endogenous Cushing's syndrome
}

\author{
Irene Zervolea, Harris Pratsinis, Stylianos Tsagarakis ${ }^{1}$, Niki Karavitaki ${ }^{1}$, Dimitri Stathakos, Nikos Thalassinos ${ }^{1}$ \\ and Dimitris Kletsas \\ Laboratory of Cell Proliferation and Ageing, Institute of Biology, National Centre for Scientific Research 'Demokritos', 15310 Athens, Greece and \\ ${ }^{1}$ Department of Endocrinology, Diabetes and Metabolism, 'Evangelismos' Hospital, Athens, Greece \\ (Correspondence should be addressed to D Kletsas; Email: dkletsas@bio.demokritos.gr)
}

(I Zervolea and M Pratsinis contributed equally to this work and S Tsagarakis and D Kletsas share senior authorship)

\begin{abstract}
Objective: Chronic exposure to elevated glucocorticoid (GC) concentrations induces detrimental effects in several tissues. In the skin, GCs provoke intense alterations on various parameters of the physiology of fibroblasts, cumulatively leading to skin atrophy and impaired wound healing. As there are concerns that GCs may generate permanent adverse functional changes, we have investigated whether chronic in vivo exposure to GC excess results in persisting defects in skin fibroblasts.

Design and methods: We have studied in vitro primary skin fibroblast cultures obtained from patients suffering from endogenous Cushing's syndrome (CF), as well as from sex- and age-matched normal donors (NF). The following functional parameters were investigated: cell proliferation, secretion of collagen, matrix metalloproteinases (MMPs) and their inhibitors (tissue inhibitors of metalloproteinases; TIMPs) and contractile capacity.

Results: CFs, grown under standard culture conditions in the absence of a hypercortisolemic milieu, exhibited an increased proliferative capacity and a higher final cell culture density compared with NFs. Collagen synthesis, in the absence or presence of transforming growth factor- $\beta$, was equal to that of NFs. However, CFs secreted comparatively lower levels of MMP-1, MMP-2 and TIMP-1, and nearly equal levels of TIMP-2. CFs also exhibited an increased ability to contract gels of polymerized collagen.

Conclusions: Collectively, these functional characteristics of CFs are in contrast to the known catabolic effects of GCs, and suggest that prior exposure to GC excess is not associated with a persisting adverse outcome in the functional phenotype of the fibroblasts.
\end{abstract}

European Journal of Endocrinology 152 895-902

\section{Introduction}

Chronic glucocorticoid (GC) excess, either exogenous, as in the course of corticosteroid therapy, or endogenous, as in the various forms of Cushing's syndrome, has severe detrimental effects in almost all tissues, including skin, bones, muscles and brain $(1,2)$. Although on clinical grounds there seems to be an improvement in tissue damage following restoration of a normal GC milieu, there are concerns that recovery may be incomplete due to permanent cellular defects from prior GC excess (3-5). In addition, it has been suggested that GCs may accelerate the aging process (5). The skin is one of the tissues most profoundly affected by GC excess, leading to atrophy and impaired wound healing $(2,6,7)$. The detrimental effects of GCs, as shown by in vivo and in vitro studies, are mediated primarily by interferences with the function of skin fibroblasts. In particular, GCs induce profound alterations on several parameters of extracellular matrix (ECM) homeostasis, such as a decrease in collagen synthesis and an altered expression of matrix metalloproteinases (MMPs) and their inhibitors (tissue inhibitors of metalloproteinases; TIMPs), leading finally to a reduction of the collagen mass $(8-10)$, while controversial data have been reported on their effect on the regulation of fibroblast proliferation (11).

Endogenous Cushing's syndrome is a rare disease due to either adrenocorticotropin (ACTH)-independent or ACTH-dependent cortisol overproduction by the adrenal glands (12). At the time of diagnosis most patients have atrophic skin and impaired wound healing. Skin fibroblasts from these individuals therefore 
represent a model suitable for the investigation in vitro of the cumulative effects of prior GC exposure and in particular of the ability of GCs to produce permanent changes in cellular function that may adversely affect tissue homeostasis. To this purpose, in the present study we have developed primary cultures of skin fibroblasts obtained from patients suffering from endogenous Cushing's syndrome, as well as from sex- and age-matched normal donors, and have studied their functional characteristics when grown under standard culture conditions in vitro in the absence of exogenous GC administration. Specifically, we have investigated possible long-lasting alterations concerning central fibroblast activities involved in skin homeostasis and wound repair, such as proliferation and contraction, as well as production and degradation of extracellular matrix components.

\section{Materials and methods}

\section{Materials}

Cell culture media were purchased from Seromed (Berlin, Germany); fetal bovine serum (FBS) was from Gibco BRL (Paisley, Strathclyde,UK); [methyl- $\left.{ }^{3} \mathrm{H}\right]$ thymidine and $\mathrm{L}-\left[{ }^{3} \mathrm{H}\right]$ proline were from Amersham International plc (Amersham, Bucks, UK). Human recombinant transforming growth factor (TGF)- $\beta 1$ was purchased from R \& D Systems (Minneapolis, MN, USA). Polyclonal antibodies against TIMP-1 and TIMP-2 were from Sigma-Aldrich (St Louis, MO, USA) and that against MMP-1 was from NeoMarkers (Lab Vision Corporation, Fremont, CA, USA). Cell culture plastics were obtained from Corning Bibby Sterilin (New York, NY, USA). All other chemicals were from Sigma-Aldrich.

\section{Cells and cell culture conditions}

Fibroblast cultures were established from biopsies taken from the inner side of the left arm of six patients suffering from active Cushing's syndrome, being between 38 and 54 years of age (Cushing's fibroblasts; CFs), as well as from six age- and sex-matched normal individuals (normal fibroblasts; NFs). The clinical characterisitics of patients with endogenous Cushing's syndrome are shown in Table 1. Written informed consent was obtained from all patients, as well as from the normal volunteers participating in this study. The study was approved by the ethical committee of Evangelismos Hospital.

Primary cultures were established from skin explants, and cells were grown in Earle's minimum essential medium (MEM) supplemented with antibiotics, nonessential amino acids and 15\% FBS. When the primary cultures were confluent, they were designated as being in passage 1 , and they were subcultured by using trypsin-citrate $(0.25-0.3 \%(\mathrm{w} / \mathrm{v}))$ solution at a $1: 2$ split ratio (13). From this point on, serial passaging was performed once a week. In all experiments, fibroblast cultures up to passage 5 were used.

\section{Collagen synthesis}

Collagen synthesis was measured by a modification of the protease-free collagenase method (14). Fibroblast cultures, after reaching confluency, were arrested by culturing for $48 \mathrm{~h}$ in MEM containing $0.1 \%$ FBS. The medium was then removed and cultures were supplemented with fresh medium, containing $5 \mu \mathrm{Ci} / \mathrm{ml}$ $\mathrm{L}-\left[{ }^{3} \mathrm{H}\right]$ proline $(126 \mathrm{mCi} / \mathrm{mmol}), \quad \beta$-aminopropionitrile $(\beta A P N ; 50 \mu \mathrm{g} / \mathrm{ml})$ and ascorbic acid $(50 \mu \mathrm{g} / \mathrm{ml})$, and incubated for a further $48 \mathrm{~h}$. The culture supernatant was collected and proteins were precipitated with trichloroacetic acid (TCA; final concentration 10\%). The resulting pellet was dissolved in $\mathrm{NaOH}(0.2 \mathrm{M})$, and one half of each sample was digested with protease-free collagenase $(5$ bovine tendon collagen units $/ \mathrm{ml}$ buffer containing $0.005 \mathrm{M} \mathrm{CaCl}_{2}, 0.2 \mathrm{M}$ $\mathrm{NaCl}$ and $0.05 \mathrm{M}$ Tris $-\mathrm{HCl}(\mathrm{pH} 7.4))$ for $2 \mathrm{~h}$ at $37^{\circ} \mathrm{C}$ and $16 \mathrm{~h}$ at room temperature; subsequently, the undigested protein was precipitated with TCA. After centrifugation, the supernatant and one wash were collected and radioactivity was measured in a $\beta$-counter. Collagen synthesis of each sample was calculated by subtracting the radioactivity of the blank (untreated half of the sample) from the radioactivity of the collagenase-treated half. Finally, the results were normalized to the number of cells recovered (15). It must be noted that preliminary experiments have shown that approximately $95 \%$ of the collagen produced is secreted in the culture milieu.

Table 1 Clinical characteristics of patients with endogenous Cushing's syndrome.

\begin{tabular}{lccccc}
\hline Patient & Age (years) & Diagnosis & UFC $(\mu \mathrm{g} / \mathbf{2 4 h})$ & Skin involvement & Estimated duration of disease (years) \\
\hline 1 & 38 & CD & 200 & Yes & 5 \\
2 & 52 & AA & 203 & Yes & 3 \\
3 & 44 & AA & 486 & Yes & 5 \\
4 & 54 & CD & 609 & Yes & 2 \\
5 & 26 & CD & 936 & Yes & 2 \\
6 & 40 & CD & 378 & 4 & \\
\hline
\end{tabular}

Diagnosis was histologically confirmed in all patients: CD, Cushing's disease; AA, adrenal adenoma. UFC, urinary free free cortisol. Skin involvement includes atrophy, bruises and striae. Estimated duration of disease was based on disease-specific symptoms and serial photographs when available. 


\section{Zymography}

Gelatin zymography was performed using a modification of the procedure of Herron et al. (16), as follows. Cells were grown to confluency and after extensive washing they were incubated for $48 \mathrm{~h}$ in serum-free medium. Conditioned media were then collected and separated under non-reducing conditions on 10\% SDS polyacrylamide gels, impregnated with $1 \mathrm{mg} / \mathrm{ml}$ gelatin. After electrophoresis, SDS was eluted from the gels by shaking in a buffer $(5 \mathrm{mM} \mathrm{CaCl} 2$ and $50 \mathrm{mM}$ Tris-HCl, $\mathrm{pH}$ 7.4) containing $2.5 \%$ Triton-X-100, three times for $20 \mathrm{~min}$ at room temperature. The gels were then incubated for $40 \mathrm{~h}$ at $37^{\circ} \mathrm{C}$ in a substrate buffer $\left(5 \mathrm{mM} \mathrm{CaCl}_{2}\right.$ and $50 \mathrm{mM}$ Tris- $\mathrm{HCl}$, pH 7.4). The gels were stained with Coomasie Brilliant Blue R250, and gelatin-degrading enzymes were identified as clear bands against a blue background (15). The intensity of the bands was quantified using a VilberLourmat Image Analyzer (Torcy-Paris, France) and the BioProfil software (Vilber-Lourmat, Torcy-Paris, France) and the results were normalized according to the cell number of each culture.

\section{Western analysis}

Conditioned media, collected as described above, were concentrated 20-fold by TCA precipitation and resuspension in serum-free medium (for TIMP analysis) or by Vivaspin concentrators (MWCO $10 \mathrm{kDa}$; Sartorius AG, Goettingen, Germany) (for MMP-1 analysis). They were then electrophoresed under reducing conditions on $10 \%$ SDS polyacrylamide gels, and the proteins were transferred to polyvinylidene difluoride membranes (Amersham). The membranes were blocked overnight with $5 \%$ non-fat milk in $150 \mathrm{mM}$ $\mathrm{NaCl}$, in $10 \mathrm{mM}$ Tris- $\mathrm{HCl}$ buffer, $\mathrm{pH}$ 7.4, containing $0.05 \%$ Tween-20 and incubated with the primary antibodies for $1 \mathrm{~h}$ and then with horseradish peroxidaseconjugated second antibody $(1 \mathrm{~h})$. Immunoreactive bands were visualized on Kodak-X-OMAT AR film by chemiluminescence (ECL kit) according to the manufacturer's (Amersham) instructions. Densitometric analysis was performed as above.

\section{DNA synthesis}

Cells were plated in MEM supplemented with 10\% FBS at a density of 10000 cells $/ \mathrm{cm}^{2}$ and $24 \mathrm{~h}$ later cell counting was performed (see below), in order to determine the plating efficiency. In parallel cultures, the medium was changed to a fresh one containing [methyl- ${ }^{3} \mathrm{H}$ ]thymidine $\quad(0.15 \mu \mathrm{Ci} / \mathrm{ml}, \quad 25 \mathrm{Ci} / \mathrm{mmol})$. After further incubation for $20 \mathrm{~h}$, the radioactivity incorporated in DNA was counted, as previously described (17). Briefly, the cells were fixed with TCA $(10 \%(\mathrm{w} / \mathrm{v}))$, solubilized with $1 \%$ SDS/0.3 M NaOH, added to a scintillation cocktail and subjected to scintillation counting (18).

\section{Determination of the proliferative capacity of the fibroblasts}

Cells were plated in MEM containing 10\% FBS at a density of 10000 cells $/ \mathrm{cm}^{2}$ and were grown with medium changes every second day. Cells were detached, suspended in culture medium and counted, by means of a Coulter counter, at 24 and $72 \mathrm{~h}$ after plating, while cultures were still at their exponential phase of growth. The doubling time (DT) of each culture was estimated using the cell numbers at $24 \mathrm{~h} \mathrm{(n1)}$ and $72 \mathrm{~h}(\mathrm{n} 2)$, as follows: DT $(\mathrm{h})=48 /[(\operatorname{logn} 2-\operatorname{logn} 1) / \log 2]$. All measurements were performed in triplicate.

\section{Gel contraction}

Collagen matrix contraction assay was performed as follows. Hydrated collagen matrices were prepared by mixing a rat-tail collagen stock solution $(3 \mathrm{mg} / \mathrm{ml})$ with sodium bicarbonate $(0.15 \mathrm{M})$ and MEM $(10 \times)$, at a ratio of $8: 2: 1$. One milliliter of collagen solution, populated with $2 \times 10^{5}$ cells, was added to each well of a 12-well plate and matrices were left to polymerize at $37^{\circ} \mathrm{C}$, in the presence of MEM containing $10 \%$ FBS. Forty-eight hours later, the matrices were gently released from the underlying culture dish by using a spatula and then incubated at $37^{\circ} \mathrm{C}$ for another $3 \mathrm{~h}$. To determine the extent of contraction, matrices were fixed in 3\% paraformaldehyde in phosphate-buffered saline for $10 \mathrm{~min}$. The matrices were washed, placed on a flat surface and measured with a ruler. Contraction is represented as the diameter of the contracted matrix as a percentage of the initial gel.

\section{Statistics}

Results are presented as average values derived from three independent experiments. The two groups of fibroblast strains (CF and NF) are compared based on the median values $( \pm$ S.E. $)$ for each group.

Due to the small number of cell strains, the differences between the two groups were evaluated by using a non-parametric test (Mann and Whitney rank sum test).

\section{Results}

\section{Regulation of collagen synthesis and degradation in CRs}

First, collagen production was studied by measuring the incorporation of $\left[{ }^{3} \mathrm{H}\right]$ proline into newly secreted collagen. Normalization of proline incorporation to the number of cells in culture showed that CFs secreted near equal amounts of collagen in comparison with 
normal cells (median values: 4751土 383 c.p.m. $/ 10^{5}$ cells vs $4240 \pm 417$ c.p.m. $/ 10^{5}$ cells, $P=0.631$ ) (Fig. 1A). This result was in accordance with the fact that all $\mathrm{CF}$ and NF cultures expressed nearly equal amounts of $\alpha 1$-procollagen mRNA, as assessed by RT-PCR analysis (not shown here). Furthermore, when treated with TGF- $\beta$ - a potent inducer of collagen synthesis - NFs and CFs exhibited similar stimulation of $\left[{ }^{3} \mathrm{H}\right]$ proline incorporation over control levels (Fig. 1B).

Collagen accumulation in the tissue is not determined solely by the degree of its synthesis but also by its degradation, the latter being regulated by the relative levels of the secreted matrix MMPs and their inhibitors (TIMPs). Thus, serum-free conditioned

(A)

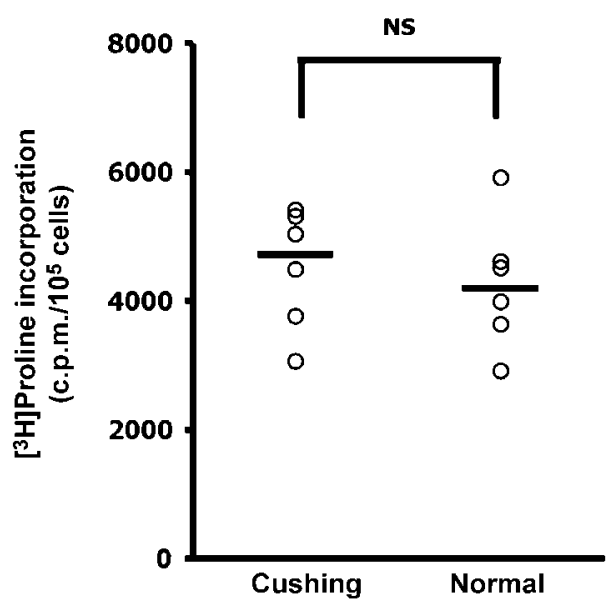

(B)

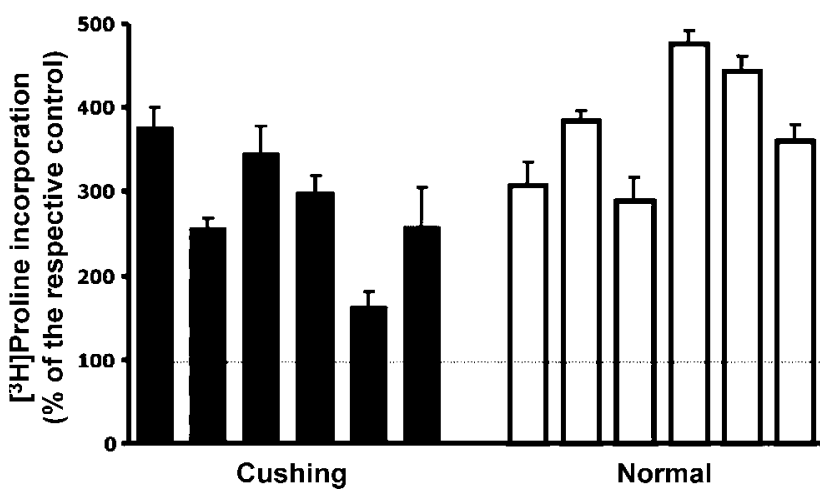

Figure 1 Collagen synthesis by CFs and NFs. Confluent cultures of CFs and NFs were incubated for $48 \mathrm{~h}$ in the presence of MEM supplemented with $0.1 \%$ FBS. Then, fresh medium, with or without TGF- $\beta 1(2 \mathrm{ng} / \mathrm{ml})$, was added along with $\left[{ }^{3} \mathrm{H}\right]$ proline $(5 \mu \mathrm{Ci} / \mathrm{ml}), \beta A P N(50 \mu \mathrm{g} / \mathrm{ml})$ and ascorbic acid $(25 \mu \mathrm{g} / \mathrm{ml})$ and after another $48 \mathrm{~h}$ of incubation collagen synthesis was estimated, as described in Materials and methods. In (A) the values correspond to basal (unstimulated) collagen synthesis (the horizontal bars indicate the median value for each group, i.e. CFs and NFs; NS: non-significant). In (B) the values represent percent stimulation of the TGF- $\beta$-treated culture in comparison with the untreated one (error bars indicate S.D.). media from all fibroblast cultures were collected, concentrated, electrophoresed and subjected to Western analysis, by using specific antibodies against MMP-1, TIMP-1 and TIMP-2. MMP-2 in particular was studied by gelatin zymography. Western analysis for MMP-1 and zymography for MMP-2 demonstrated only the presence of the latent forms of these MMPs. On average, CFs secreted lower levels of MMPs and TIMPs (Fig. 2). In particular, densitometric analysis revealed a lower secretion of MMP-1 $(30.4 \pm 4.6$ arbitrary units (AU) for CFs vs $55.4 \pm 9.4$ for NFs, $P=0.010$ ), MMP-2 $(61.8 \pm 2.4 \mathrm{AU}$ for $\mathrm{CFs}$ vs $91.3 \pm 5.3$ for NFs, $P=0.004)$ and TIMP-1 $(28.3 \pm 5.4 \mathrm{AU}$ for $\mathrm{CFs}$ vs $56.4 \pm 11.4$ for NFs, $P=0.037$ ). On the other hand, TIMP-2 levels were nearly equal for both cell types $(67.7 \pm 8.9 \mathrm{AU}$ for $\mathrm{CFs}$ vs $76.5 \pm 9.2$ for $\mathrm{NFs}$, $P=0.749)$.

\section{The proliferative capacity of CFs}

As can be seen in Table 2, CF cultures proliferated faster than NF, as revealed by the incorporation of radiolabeled thymidine (median values: $325 \pm 49$ c.p.m. $/ 10^{3}$ cells for CFs vs $210 \pm 39$ c.p.m. $/ 10^{3}$ cells for NFs, $P=0.037)$. In parallel, CF strains tended to demonstrate shorter doubling times than NF ones, although the median difference was marginally statistically non-significant $\quad(28.3 \pm 1.5 \mathrm{~h} \quad$ vs $\quad 33.9 \pm 1.8 \mathrm{~h}$, $P=0.055) \quad$ (Table 2). Interestingly, CFs exhibited much higher final cell densities than fibroblasts from

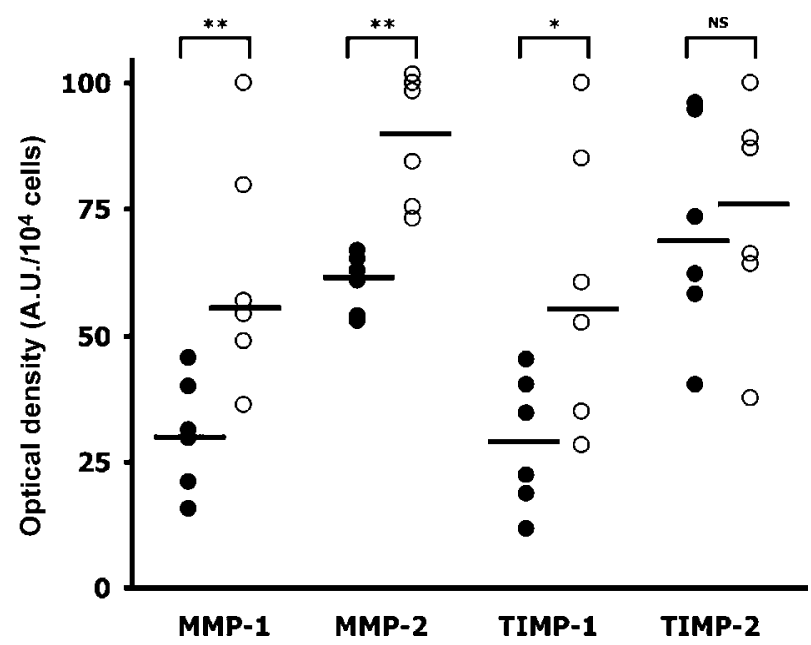

Figure 2 Secretion of MMPs and TIMPs by CFs $(\bullet)$ and NFs $(O)$. Serum-free media conditioned for $48 \mathrm{~h}$ by confluent cultures of arrested CFs or NFs were concentrated 20 -fold, electrophoresed and subjected to Western analysis for the determination of MMP-1, TIMP-1 and TIMP-2 levels; alternatively, they were subjected to gelatin zymography without prior concentration for the detection of MMP-2. After densitometric analysis, the values were normalized to the number of cells recovered. The horizontal bars indicate the median value for each group. ${ }^{\star} P<0.05$, ${ }^{\star} P<0.01$, NS: non-significant. 
Table 2 Rate of proliferation of CFs vs NFs.

\begin{tabular}{|c|c|c|c|c|c|}
\hline \multicolumn{3}{|c|}{ CFs } & \multicolumn{3}{|c|}{ NFs } \\
\hline & c.p.m. $/ 10^{3}$ cells & Doubling time $(\mathrm{h})$ & & c.p.m. $/ 10^{3}$ cells & $\overline{\text { Doubling time }(\mathrm{h})}$ \\
\hline CF1 & $586 \pm 47$ & $22.9 \pm 0.1$ & NF1 & $325 \pm 16$ & $31.3 \pm 0.6$ \\
\hline CF2 & $275 \pm 61$ & $28.2 \pm 0.3$ & NF2 & $75 \pm 1$ & $38.2 \pm 0.6$ \\
\hline CF3 & $336 \pm 80$ & $30.0 \pm 1.0$ & NF3 & $146 \pm 40$ & $30.1 \pm 0.9$ \\
\hline CF4 & $314 \pm 84$ & $33.6 \pm 0.4$ & NF4 & $189 \pm 10$ & $36.4 \pm 0.5$ \\
\hline CF5 & $442 \pm 3$ & $25.8 \pm 2.6$ & NF5 & $230 \pm 6$ & $37.2 \pm 0.2$ \\
\hline CF6 & $292 \pm 70$ & $28.4 \pm 1.0$ & NF6 & $307 \pm 36$ & $27.3 \pm 0.5$ \\
\hline Median & $325 \pm 49$ & $28.3 \pm 1.5$ & Median & $210 \pm 39$ & $33.9 \pm 1.8$ \\
\hline
\end{tabular}

DNA synthesis rate of CF vs NF culures in the presence of $10 \%$ FBS, as well as their doubling time rate, were determined as described in Materials and methods. The average of three independent experiments for each cell strain is presented ( \pm S.D.), while at the bottom of the Table the median of each group is indicated ( \pm S.E. $)$.

normal donors, after culture for 12 days in the presence of $10 \%$ FBS. The median cell density was $114500 \pm 12700$ cells $/ \mathrm{cm}^{2}$, i.e. approximately $40 \%$ higher compared with that of NFs (78 500 \pm 7000 cells $/ \mathrm{cm}^{2}, P=0.016$ ) (Fig. 3).

\section{Increased ability of CFs for gel contraction}

Contraction represents an important fibroblast function in the wound-healing process. Having in mind that Cushing's patients are characterized by impaired tissue repair capacity, we have also studied the ability of fibroblasts from these patients to contract gels of polymerized collagen.

Accordingly we have developed collagen matrices populated by an equal number of $\mathrm{CFs}$ and NFs

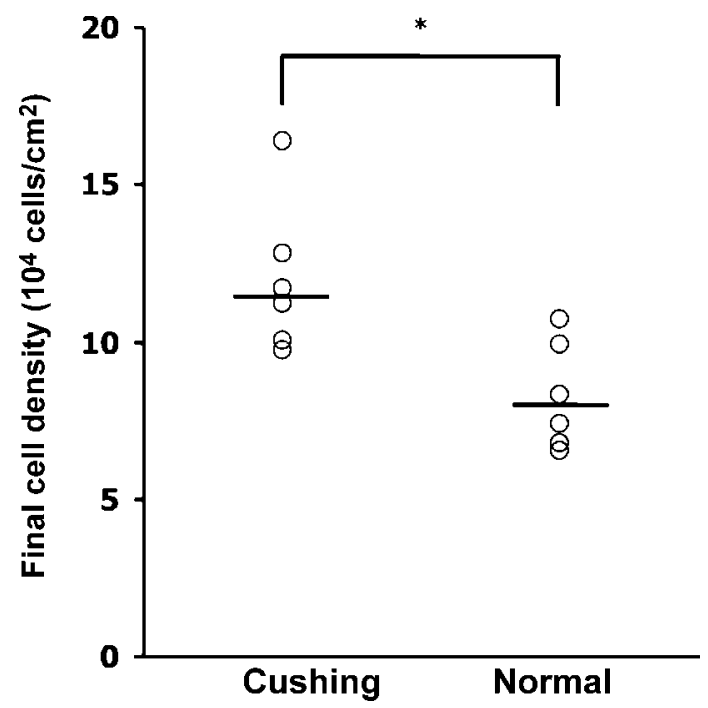

Figure 3 Final cell density of CF and NF cultures. The cell density achieved by the established fibroblast cultures after 12 days of growth in the presence of $10 \%$ FBS was determined as described in Materials and methods. Each point represents the average final density for each cell strain as determined after three independent experiments. The horizontal bars indicate the median value for each group. ${ }^{*} P<0.05$.
(300 000 cells $/ \mathrm{ml}$ gel). After polymerization, the cells were left unstimulated for 2 days. They were then released from the plastic dish and contraction was measured $3 \mathrm{~h}$ later. As shown in Fig. 4, CFs exhibited an increased ability for gel contraction, as compared with control fibroblasts (percentage of the initial diameter: $46.0 \pm 1.1$ for CFs vs $54.8 \pm 3.1$ for NFs, $P=0.027)$.

\section{Discussion}

The aim of the present study was to investigate whether chronic in vivo exposure to GC excess results in persisting defects of skin fibroblasts. To that end, we have tested in vitro the functional characteristics of fibroblasts from patients with endogenous Cushing's

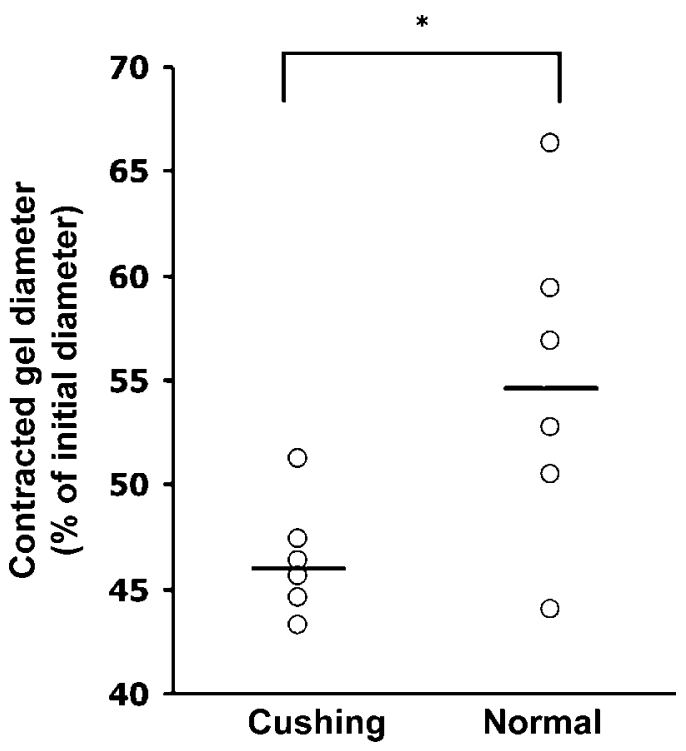

Figure 4 Collagen gel contraction by CFs vs NFs. Floating collagen gels populated by CFs or NFs were left to contract for $3 \mathrm{~h}$. Values, representing percent contraction of the gel in comparison with the initial non-contracted one, are the mean of three independent experiments performed in triplicate. The horizontal bars indicate the median value for each group. ${ }^{\star} P<0.05$. 
syndrome. The advantage of studying patients with endogenous GC excess is that they are devoid of the disease-related abnormalities requiring exogenous administration of GC therapy. We have found that collagen synthesis, which is most profoundly affected during GC excess, was comparable with normal following the removal of skin fibroblasts from the high GC milieu of Cushing's syndrome patients. Moreover, skin fibroblasts from these patients exhibited an increased proliferative capacity in culture, a higher final cell culture density and an increased contractile ability. Collectively, these anabolic responses were in clear contrast to the catabolic effects of GCs observed in vivo or in vitro and indicate that prior chronic exposure to GC excess is not associated with a persisting adverse outcome in the functional phenotype of these cells. The in vivo situation in patients with endogenous Cushing's syndrome involves exposure of these cells in higher (but not pharmacological) cortisol concentrations for long timeintervals (years). Patients with exogenous syndrome due to GC administration are usually exposed to a different pattern of GC excess. Therefore, the data presented by this study may not necessarily be extrapolated to exogenous Cushing's syndrome.

Collagen is the most abundant protein in the dermis and consequently inhibition of its synthesis could be the primary cause of the dermal atrophy observed in Cushing's syndrome patients. Furthermore, a shift of the balance between MMPs and their inhibitors (TIMPs) towards collagenolysis could play an equally important role in the deregulation of skin homeostasis. In fact, it is well recognized that acute in vivo and in vitro exposure to GCs grossly diminished type I procollagen synthesis at the mRNA and protein level in human dermal fibroblasts $(8,19)$. Repression of procollagen genes has been attributed to a co-ordinate action of GCs through both GC- and TGF- $\beta$-responsive elements (20). On the other hand, in previous studies, expression of MMP-1 and -2 was found to be reduced following treatment with GCs $(8,21)$. Furthermore, GCs were found to decrease TIMP-2 production. With regard to TIMP-1, although the existing data are still controversial, the majority of reports point towards a decreased synthesis $(8,21,22)$. In our experiments, however, skin fibroblasts from Cushing's syndrome patients, when removed from the high cortisol milieu in vivo and cultured in vitro in the absence of GCs, restored their ability to express $\alpha 1$-procollagen mRNA, compared with cells from normal donors (data not shown). With regard to the MMP/TIMP balance, we found that CFs secreted lower levels of MMP-1, MMP-2 and TIMP-1 compared with normal cells, while both cell types secreted nearly equal amount of TIMP-2. Thus, changes in MMPs and TIMPs seem to persist following prior GC exposure. Despite this, $\left[{ }^{3} \mathrm{H}\right]$ proline incorporation either basal or TGF- $\beta 1$-stimulated - estimated by the protease-free collagenase method, was almost identical between fibroblasts derived from Cushing's patients and normal volunteers. This assay measures the net effect of novel collagen synthesis and secretion, as well as of its degradation by the secreted collagenases, and represents a good approximation of collagen accumulation. It is therefore suggested that the observed changes in MMPs and TIMPs do not adversely affect ECM homeostasis.

In addition, our studies revealed that CFs possess a significantly increased proliferative ability compared with normal donor cells, expressed either as stimulation of DNA synthesis or as decreased population doubling time. It should be noted that the data on the effect of GCs on the proliferation of human fibroblasts in vitro are still controversial. Indeed, their effect has been reported to extend from inhibitory to stimulatory (2326). This has been attributed to the type of GC or the culture conditions used (11). However, all the above refer to studies focused on the acute effect of GCs on cellular proliferation. On the other hand, it has been reported that in vitro long-term treatment of human embryonic lung fibroblasts with cortisol enhanced the proliferative activity of these cells (27). This is in clear agreement with the data reported here, where it seems that the in vivo long-term exposure to high levels of cortisol in Cushing's syndrome patients results in an increased proliferative ability of skin fibroblasts.

One of the most striking differences observed between CF and NF cultures was their final cell density at confluence. The mean saturation density in CF culture was approximately $40 \%$ higher than that found in the NF cultures. It should be noted that previous in vitro data indicated that exogenous addition of GCs could increase the final cell density in various strains of normal fibroblasts; interestingly, this increase has been reported to range between 50 and $70 \%(25,28,29)$, i.e. similar to that observed here for CFs. This phenomenon is most likely due to the above-mentioned high proliferative potential of CFs.

Fibroblast-mediated contraction is an important parameter of skin homeostasis. Gels of polymerized collagen populated by fibroblasts provide a functional in vitro model of tissue contraction (30). GCs, such as dexamethasone or hydrocortisone, can reduce skin fibroblast-mediated contraction, and this effect may play a role in the delay of the wound-healing process $(31,32)$. This inhibition is reversed when the GCs are removed but, while the effect of hydrocortisone is entirely reversible, that of dexamethasone is not (32). Here we report that when the fibroblasts of Cushing's syndrome patients were cultured in vitro in the absence of GCs their contractile ability was not only restored, but they further exhibited a significantly increased capacity for collagen gel contraction, compared with cells from normal donors.

On the whole, CFs after their removal from the tissue and under standard culture conditions exhibit an increased proliferative capacity, a higher final cell density and an augmented contractile ability, while 
the collagen production is restored. Collectively, these anabolic responses are in clear contrast to the catabolic effects of GCs, and can possibly represent a typical 'rebound' reaction, analogous to that observed in cells of immune origin (33). It should be also noted that our findings are not in accordance with previous suggestions that GCs may induce age-related alterations in skin fibroblasts. Senescent fibroblasts are characterized by a decreased proliferation and lower final density (18), decreased contractility (34) and an increased collegenolytic activity (35). All these changes are clearly in an opposite direction from the functional phenotype of CFs reported here. In this context, and in agreement with the data presented here, we have recently shown that CFs exhibited a remarkable extension of their in vitro lifespan (36).

In conclusion, our results indicate that long-term exposure to high GC concentrations in vivo is not associated with persisting adverse changes in skin fibroblasts and/or acceleration of the aging process of these cells. Instead, previous long-lasting exposure to the conditions of the plasma of Cushing's patients has an unexpected positive effect on the physiology of these cells when cultured in vitro.

\section{Acknowledgements}

We thank Dr A Aletras for providing the MMP-1 antibody. The technical assistance of Mrs E Sevaslidou is gratefully acknowledged. This work was partly supported by the European Union (contract No. QLK6CT-2002-02 582) and by postdoctoral fellowships from the Greek State Scholarship's Foundation (IKY) to $\mathrm{H} \mathrm{P}$ and $\mathrm{I} \mathrm{Z}$.

\section{References}

1 Boumpas DT, Chrousos GP, Wilder RL, Cupps TR \& Balow JE. Glucocorticoid therapy for immune-mediated diseases: basic and clinical correlates. Annals of Internal Medicine 1993119 1198-1208.

2 Boscaro M, Barzon L, Fallo F \& Sonino N. Cushing's syndrome. Lancet $2001357783-791$.

3 Bourdeau I, Bard C, Noel B, Leclerc I, Cordeau MP, Belair M, Lesage J, Lafontaine L \& Lacroix A. Loss of brain volume in endogenous Cushing's syndrome and its reversibility after correction of hypercortisolism. Journal of Clinical Endocrinology and Metabolism 200287 1949-1954.

4 Kleerekopper M \& Schiebinger RJ. Skeletal recovery after treatment of Cushing's: still room for improvement. Journal of Clinical Endocrinology and Metabolism $1995 \mathbf{8 0} 2856-2858$.

5 Sapolsky RM. Stress, glucocorticoids, and damage to the nervous system: the current state of confusion. Stress 19961 1-19.

6 Sowers JR \& Lippman HR. Cushing's syndrome due to ectopic ACTH production: cutaneous manifestations. Cutis $1985 \mathbf{3 6}$ $351-352,354$.

7 Oikarinen A \& Autio P. New aspects of the mechanism of corticosteroid-induced dermal atrophy. Clinical and Experimental Dermatology $199116416-419$.

8 Slavin J, Unemori E, Hunt TK \& Amento E. Transforming growth factor beta (TGF-beta) and dexamethasone have direct opposing effects on collagen metabolism in low passage human dermal fibroblasts in vitro. Growth Factors 199411 205-213.

9 Oikarinen A, Haapasaari KM, Sutinen M \& Tasanen K. The molecular basis of glucocorticoid-induced skin atrophy: topical glucocorticoid apparently decreases both collagen synthesis and the corresponding collagen mRNA level in human skin in vivo. British Journal of Dermatology $19981391106-1110$.

10 Autio P, Oikarinen A, Melkko J, Risteli J \& Risteli L. Systemic glucocorticoids decrease the synthesis of type I and type III collagen in human skin in vivo, whereas isotretinoin treatment has little effect. British Journal of Dermatology 1994131 660-663.

11 Durant S, Duval D \& Homo-Delarche F. Factors involved in the control of fibroblast proliferation by glucocorticoids: a review. Endocrine Reviews $19867254-269$.

12 Orth DN. Cushing's syndrome. New England Journal of Medicine $1995332791-803$.

13 Kletsas D, Caselgrandi E, Barbieri D, Stathakos D, Franceschi C \& Ottaviani E. Neutral endopeptidase-24.11 (NEP) activity in human fibroblasts during development and ageing. Mechanisms of Ageing and Development $1998 \mathbf{1 0 2} 15-23$.

14 Peterkofsky B \& Diegelmann R. Use of a mixture of proteinase-free collagenases for the specific assay of radioactive collagen in the presence of other proteins. Biochemistry $197110988-994$.

15 Zervolea I, Kletsas D \& Stathakos D. Autocrine regulation of proliferation and extracellular matrix homeostasis in human fibroblasts. Biochemical and Biophysical Research Communications $2000276785-790$.

16 Herron GS, Banda MJ, Clark EJ, Gavrilovic J \& Werb Z. Secretion of metalloproteinases by stimulated capillary endothelial cells. II. Expression of collagenase and stromelysin activities is regulated by endogenous inhibitors. Journal of Biological Chemistry 1986 $2612814-2818$.

17 Kletsas D, Stathakos D, Sorrentino V \& Philipson L. The growthinhibitory block of TGF-beta is located close to the G1/S border in the cell cycle. Experimental Cell Research 1995217 477-483.

18 Kletsas D \& Stathakos D. Quiescence and proliferative response of normal human embryonic fibroblasts in homologous environment. Effect of aging. Cell Biology International Reports 199216 103-113.

19 Cockayne D, Sterling KM Jr, Shull S, Mintz KP, Illeyne S \& Cutroneo KR. Glucocorticoids decrease the synthesis of type I procollagen mRNAs. Biochemistry $1986253202-3209$.

20 Meisler N, Shull S, Xie R, Long GL, Absher M, Connolly JP \& Cutroneo KR. Glucocorticoids coordinately regulate type I collagen pro alpha 1 promoter activity through both the glucocorticoid and transforming growth factor beta response elements: a novel mechanism of glucocorticoid regulation of eukaryotic genes. Journal of Cellular Biochemistry 199559 376-388.

21 Clark SD, Kobayashi DK \& Welgus HG. Regulation of the expression of tissue inhibitor of metalloproteinases and collagenase by retinoids and glucocorticoids in human fibroblasts. Journal of Clinical Investigation $1987 \mathbf{8 0} 1280-1288$.

22 Kylmaniemi M, Oikarinen A, Oikarinen K \& Salo T. Effects of dexamethasone and cell proliferation on the expression of matrix metalloproteinases in human mucosal normal and malignant cells. Journal of Dental Research 199675 919-926.

23 Gomi M, Iida S, Tsugawa M, Itoh Y, Moriwaki K, Fujii H, Yamashita S, Tamaki T \& Tarui S. In vitro studies of skin fibroblasts from a patient with a new type of primary cortisol resistance: glucocorticoid receptor status and glucocorticoid effect on DNA synthesis. Hormone and Metabolic Research 198719 328-330.

24 Minguell JJ, Martinez J \& Walter T. Effect of hydrocortisone on the growth of human bone marrow fibroblasts. British Journal of Haematology 198252 307-310.

25 Rowe DW, Starman BJ, Fujimoto WY \& Williams RH. Differences in growth response to hydrocortisone and ascorbic acid by human diploid fibroblasts. In Vitro 197713 824-830.

26 Germinario RJ, Mcquillan A, Oliveira M \& Manuel S. Enhanced insulin stimulation of sugar transport and DNA synthesis by 
glucocorticoids in cultured human skin fibroblasts. Archives of Biochemistry and Biophysics 1983226 498-505.

27 Sluke G \& Schachtschabel DO. Long-term treatment with cortisol influence on proliferation, aging and glycosaminoglycan synthesis of cultured human diploid fibroblasts (WI-38). Zeitschrift für Gerontologie 198821 68-73.

28 Russell JD, Russell SB \& Trupin KM. Differential effects of hydrocortisone on both growth and collagen metabolism of human fibroblasts from normal and keloid tissue. Journal of Cellular Physiology $197897221-229$

29 Kondo H, Kasuga H \& Noumura T. Effects of various steroids on in vitro lifespan and cell growth of human fetal lung fibroblasts (WI38). Mechanisms of Ageing and Development 198321 335-344.

30 Grinnell F. Fibroblast-collagen-matrix contraction: growth-factor signalling and mechanical loading. Trends in Cell Biology 2000 $10362-365$.

31 Gabbiani G. The role of contractile proteins in wound healing and fibrocontractive diseases. Methods and Achievements in Experimental Pathology 1979 9 187-206.

32 Coulomb B, Dubertret L, Bell E \& Touraine R. The contractility of fibroblasts in a collagen lattice is reduced by corticosteroids. Journal of Investigative Dermatology 198482 341-344.
33 Almawi WY, Hess DA, Assi JW, Chudzik DM \& Rieder MJ. Pretreatment with glucocorticoids enhances T-cell effector function: possible implication for immune rebound accompanying glucocorticoid withdrawal. Cell Transplantation 19998 637-647.

34 Kono T, Tanii T, Furukawa M, Mizuno N, Kitajima J, Ishii M \& Hamada T. Correlation between ageing and collagen gel contractility of human fibroblasts. Acta Dermatologica Venereologica 1990 70 241-244.

35 Millis AJ, Hoyle M, McCue HM \& Martini H. Differential expression of metalloproteinase and tissue inhibitor of metalloproteinase genes in aged human fibroblasts. Experimental Cell Research $1992201373-379$.

36 Pratsinis H, Tsagarakis S, Zervolea I, Giannakopoulos F, Stathakos D, Thalassinos N \& Kletsas D. Chronic in vivo exposure to glucocorticoids prolongs cellular lifespan: the case of Cushing's syndrome-patients' fibroblasts. Experimental Gerontology 200237 $1237-1245$.

Received 29 October 2004

Accepted 16 February 2005 